due to the carelessness of employees." Before the carelessness of employees can be urged as an excuse, it must first be shown (1) that the discipline is such that the railroad has every right to expect obedience and carefulnesis in general; (2) that the proved character of the employee in any responsible post is such that the road has every right to expect faithfulness from him; and (3) that the conditions of operating are such as, on the one hand, to leave as little room as possible for disaster resulting from carelessness, and, on the other, to place no undue strain on the employee's strength and capacity.

It is gratifying to see that the reputation of these roads, with respect to their care of human life, is highly valued. This augurs well for the improvement of whatever conditions may still remain to be improved.--EDIToR.]

\section{Who Should Apply the Wassermann Test?}

To the Editor:-This week I was approached by one who styles himself a chemist asking me to instruct him in the Wassermann reaction. The followin $\because$ copy of the reply which I mailed to him is self-explanatory:

"My Dear Sir: Your inquiry yesterday, in regard to my willingness in teaching you the details of the Wassermann reaction, has received the most earnest consideration by me. Heretofore I have refused no one who wished to know this work. All such applicants, however, have been physicians.

Personally I should like to teach each one who is interested. The prevalent opinion in Germany to-day is that only trained serologists should do this work. In a recent number of the Mïnchener med. Wochenschrift, $\mathrm{K}$. Taege asserts that only his ability as a physician has enabled him to interpret difficult reactions, made by himself, satisfactorily in given cases.

To avoid being personal and prejudiced in this matter, however, I determined to place your letter in the hands of Dr. Paul G. Woolley, director of the laboratory of the Cincinnati Hospital, and to be guided by his decision. Dr. Woolley is of the opinion that I could not give you a certificate, after a period of instruction in this work, stating that you are fully competent to make a correct reading in every case which may come to you for analysis, because of your lack of medical training.

Since you asked me at the end of a period of instruction to give you a certificate stating that at such time you would be fully competent to do this work, and inasmuch as, according to the opinion of Dr. P. G. Woolley, a recognized authority in pathology, I would be unable to do so, I must refuse the offer you so kindly made me."

I am a staunch supporter of the medical profession, and I believe that none but a physician is capable of passing judgment on the question as to whether a given patient is luetic or not. Experience has shown us how difficult this reaction may become in many instances. The physicians of this state are busily engaged in trying to have the optometry bill vetoed, and I think that we all should be equally active in trying to prevent laymen from practicing medicine in any other of its branches. Furthermore no physician ought to give a certificate to any one certifying to his competency to practice any branch of medicine, and least of all in a case of this kind in which a man absolutely without a knowledge of general medicine and its ethics might affiliate himself with the advertising "venereal quack," and become a harpy to prey on the people.

This man now intends to go East with the hope of finding some physician willing to instruct him, and then to return here with a certificate announcing his fitness.

Oscar Berghausen, B.A., M.D., Cincinnati.

\section{An Observation Bearing on the Causation of Sex}

To the Editor:-I noticed in THE Journal (April 22, 1911, p. 1228) an abstract of a paper by W. W. Kain, "Can the Sex of the Family be Controlled at Will by the Parents?" Kain is said to agree with E. R. Dawson that the supplying ovary is in reality the essential factor in the causation of sex, the right ovary producing the male and the left ovary the female. I believe that a study of the chromosomes with their adjunct sex chromosomes, ete., will dispel any such notions. But that is too big a subject for this letter. I wish at this time only to report a case that came under my observation last October. A girl married at the age of 14 ; at the age of 15 she gave birth to a boy, and at 17 to a girl, following which she had three miscarriages produced by herself with a catheter. About two weeks following her last miscarriage she died of lobular pneumonia, at the age of 19. Necropsy revealed a tuberculous cavity in the right apex the size of an English walnut, a serofibrinous pleurisy of the right side, a pericarditis with effusion, no inflammation of the pleura of the left side but the pneumonic condition as bad on the left side. The pelvic area showed no evidences of the miscarriages. There was, how. ever, a congenital absence of the left tube and ovary, of which there was not even a remnant, the broad and round ligaments being apparently normal. The right ovary and tube were perfectly normal. Dr. A. E. MeDowell assisted me.

W. O. Wisner, Spokane, Wash.

\section{Queries and Minor Notes}

Anonymous Communications will not be noticed. Every letter must contain the writer's name and address, but these will be omitted, on request.

\section{VENTILATION IN THE PUBLIC SCHOOLS}

To the Editor:-I wish to get some information about ventilation of pullic schools. The system in use in our school, and in a large number of schools in New Jersey, is based on the idea that carbon dioxid is heavier than alr, which is correct at a certain temperature. The inlet for pure air is placed near the ceiling, in the expectation that the vitiated air will be forced downward to the outlet near the floor. To my mind this reverses the laws of Nature. Gases will expand when heated, volume increased, specific gravity lessened. Just imagine a class of little children seated in a kindergarten on little chairs having the impure air forced down on them in order to get it out through the outlet! When the room is heated to about $70 \mathrm{~F}$. (the transoms and windows and doors closed with this system, does the carbon dioxid rise to the ceiling? If so, why not let it out there? Being a medical inspector, I would like some light
on the subject.
Joseph PAYNe, Midland Park, N. J.

ANSwER.-You will find much information about ventilation and heating in the following books:

Shaw, $w$. N : Air Currents and the Laws of Ventilation, 1907. Cambridge Univ. Press.

Monroe, W. S.: Steam Heating and Ventilation, 1902, New ork, Engineering Record Series.

Harrington: Practical Hygiene, 1902, Lea Brothers.

You state that the inlet for pure air is near the ceiling but do not say whether the incoming air is warm or cold. The problem is one of heating as well as ventilation. If the incoming air is warm it will tend to remain near the top of the room. If the outlet is high in the wall the warm air will tend to pass out without either warming the room or materially changing the air of the room. If the outlet for air is at the floor the warm air after raising the temperature of the room will sink and pass off at the bottom.

Carbon dioxid itself in the quantity ordinarily found in rooms is harmless. It is simply taken as an indicator of vitiated air. The amount of carbon dioxid added to schoolroom air by respiration is slight, seldom more than 10 parts per 10,000. Experiments have shown that people confined in small, tight compartments endure without any symptoms of illness or discomfort an atmosphere containing 150 parts of carbon dioxid per 10,000 , provided the temperature and moisture are kept low. Tests in crowded schoolrooms under similar conditions show that the pupils are unaffected. Results are different if the temperature is allowed to rise to $70 \mathrm{~F}$. and the humidity is high. It seems well established that results commonly attributed to poor ventilation are not due to any change in chemical composition of the air but to physical changes. The solution of the problem of ventilation lies in the maintenance of the normal heat interchange between the body and the surrounding air.

Whether expired air will rise or sink depends on several factors, its temperature, amount of $\mathrm{CO}_{2}$, moisture content an.l the temperature of the surrounding air. Taking what is perhaps an average density for expired air, if the temperature of the surrounding air is below $81 \mathrm{~F}$. the expired air will tend to rise. The expired air will tend to sink if the temperature of the surrounding air is above $81 \mathrm{~F}$. and neither rise nor fall if the surrounding air is at this critical tem. perature. The expired air will sink to the floor after rising and cooling, as it will tend to do in a room temperature of $70 \mathrm{~F}$. unless the flow of air downward made by forced draught is overpowering. 